\title{
A Comparison of Discretization Methods for the Inverse Problem of Electrocardiography
}

\author{
Laura Bear ${ }^{1,2,3}$, Leo Cheng ${ }^{4}$, Rémi Dubois ${ }^{1,2,3}$, Denis Loiselle ${ }^{4}$, Bruce Smaill ${ }^{4}$ \\ ${ }^{1}$ IHU-Liryc, fondation Bordeaux Université, Pessac-Bordeaux, France. \\ ${ }^{2}$ Univ. de Bordeaux, Centre de recherche Cardio-Thoracique de Bordeaux, U1045, Bordeaux, France \\ ${ }^{3}$ INSERM, Centre de recherche Cardio-Thoracique de Bordeaux, U1045, Bordeaux, France \\ ${ }^{4}$ University of Auckland, Auckland, New Zealand
}

\begin{abstract}
Epicardial electrical activity reconstructed noninvasively from body surface potentials is currently used clinically to map electrical activity. Here, two methods for solving this problem are evaluated using in-vivo experimental data: A coupled Finite-Boundary Element Method (F-BEM) and the Method of Fundamental Solutions (MFS). Results showed similar accuracy, with a slight improvement of the MFS over the F-BEM in reconstructing ventricular electrogram magnitude, $\left(R E_{\text {mean }}=0.83 \pm 0.09\right.$ and $0.86 \pm 0.08$ respectively $)$ in the reconstructing activation maps $(C C=0.75 \pm 0.09$ and $0.73 \pm 0.12$ ) and localization of initial activation sites (LE $=19 \pm 22 \mathrm{~mm}$ and $27 \pm 22 \mathrm{~mm}$ ), though these were still not identified reliably. No improvement was seen with the inclusion of inhomogeneous structures in the F-BEM models ( $p>0.05)$. With the additional advantages in ease of implementation, the MFS is the preferred approach in this case.
\end{abstract}

\section{Introduction}

Epicardial electrical activity can be reconstructed noninvasively from body surface potentials by solving the inverse problem of electrocardiography. This approach is currently used clinically to map electrical activity and aid identification of ablation sites to alleviate ventricular tachycardia [1] and persistent atrial fibrillation [2].

There are multiple methods available to solve the inverse problem, including the commonly used Method of Fundamental Solutions (MFS) [3] and a coupled Finite/Boundary Element Method (F-BEM) [4]. In addition, inverse mapping tools in clinical use typically treat the torso as a uniform, isotropic volume conductor $[1,2]$. As the inverse problem is ill-posed, it is important to establish the limitations of each method and the extent to which lack of precision of the torso model impacts inverse mapping in-vivo. Studies have shown that body surface potentials reconstructed from epicardial potentials using homogeneous transfer matrices differ markedly from the potential distributions measured on the body surface $[5,6]$. These differences are reduced, but not eliminated by the inclusion of inhomogeneous torso electrical properties [6]. The primary objective of this study was first to evaluate the accuracy of the MFS and F-BEM approaches to inverse epicardial mapping. Second, we aimed to determine the combined effects on inverse solutions of incorporating more realistic electrical properties for skeletal muscle, fat and the lungs.

\section{Methods}

\subsection{Data sets}

In-vivo Porcine Experiments

Experimental data were obtained from anesthetized, closed-chest, pigs $(\mathrm{n}=5,30-40 \mathrm{~kg})$ [6]. Epicardial and torso potentials were recorded simultaneously using an elastic "sock" (239 unipolar electrodes, Auckland Uniservices Ltd, New Zealand) and flexible strips attached to the body surface (184 electrodes, BioSemi, the Netherlands). For each pig, recordings were made during sinus rhythm, and pacing left and right endo- , and epicardium sites. Overall, 70 records were obtained. Upon completion, the heart was arrested and MRI performed. The heart was excised and perfusion-fixed. Epicardial electrode locations were captured with a multiaxis digitizing arm (FARO Technologies, FL). MRI contrast markers placed on the "sock" and body surface strips were localized in the MR images and used to register electrode locations.

\subsection{Inverse problem formulation}

The MFS and the F-BEM were used to solve Laplace's equation, which computes the electrical potential field in the source-free volume between the heart and body 
surfaces. With the F-BEM, two heart-torso models were customized from experiment-specific MRI for each animal [6]; a homogeneous model (F-BEM homog $_{\text {) }}$ assuming isotropic conductivity, and an inhomogeneous

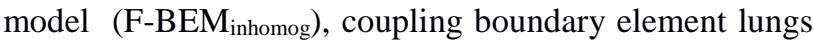
and torso cavity (assumed isotropic) to finite element fat (assumed isotropic) and skeletal muscle regions (assumed anisotropic) [4]. Kriging interpolation was used to map potentials from electrodes to the refined torso surface.

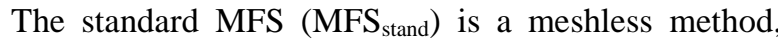
reconstructing epicardial potentials directly from torso electrode potentials assuming a homogeneous torso [3]. In order to ensure any differences seen were not due to interpolation, a fourth model was employed, using interpolated torso signals as the inputs to an MFS defined problem $\left(\mathrm{MFS}_{\mathrm{Full}}\right)$. For each of these four models, epicardial potentials were reconstructed using Tikhonov regularization and CRESO criteria.

\subsection{Data analysis}

Data analysis was performed on one representative beat for each record. Activation times (ATs) were estimated by fitting global activation fields to maximum derivative estimates and activation delays between adjacent electrograms [7]. Correspondence between measured and reconstructed electrograms (EGMs) was quantified using the relative error (RE) and correlation coefficient (CC):

$$
\begin{gathered}
R E=\left\|\emptyset_{M}-\emptyset_{R}\right\|_{2} /\left\|\emptyset_{M}\right\|_{2} \\
C C=\frac{\sum_{N}\left(\emptyset_{M}-\mu_{M}\right)\left(\emptyset_{R}-\mu_{R}\right)}{\left\|\emptyset_{M}-\mu_{M}\right\|_{2}\left\|\emptyset_{R}-\mu_{R}\right\|_{2}}
\end{gathered}
$$

where $\mathrm{N}$ is the time instant, $\emptyset_{M}^{i}$ and $\emptyset_{S}^{i}$ are the potentials at each time for measured (M) and reconstructed (R) data, while $\mu_{M}$ and $\mu_{R}$ are corresponding mean values across $\mathrm{N}$. The correspondence of measured and reconstructed ATs was also compared by evaluating RE and CC (N being the number of electrodes). ATs were also evaluated using the error in localization of earliest activation (LE), and the difference in total activation duration (ADd).

The data were analyzed using the General Linear Model facility of SAS (Statistical Analysis System). For each metric, the significance of differences among the means of the inverse methods were examined using a Nested Factorial ANOVA. The factors were "animal” and "sequence type" ('sinus rhythm', 'endocardial pacing' and 'epicardial pacing'). "Pacing locations" were nested within both factors. The resulting 8 different variance terms completely accounted for the total variance of the data. Error terms (denominators of the F-ratio) were given by higher-order interactions. When an ANOVA returned a value of $p<0.05$, then the source of the difference among means was sought using a matrix of mutuallyorthogonal contrast vectors.

\section{Results}

\subsection{Epicardial electrograms}

In Fig 1, measured and reconstructed epicardial EGMs are compared for 3 representative electrodes for a RV epicardial pacing sequence. Corresponding boxplots of $\mathrm{RE}$ and CC across all electrodes for this cardiac sequence are presented in Fig 2.

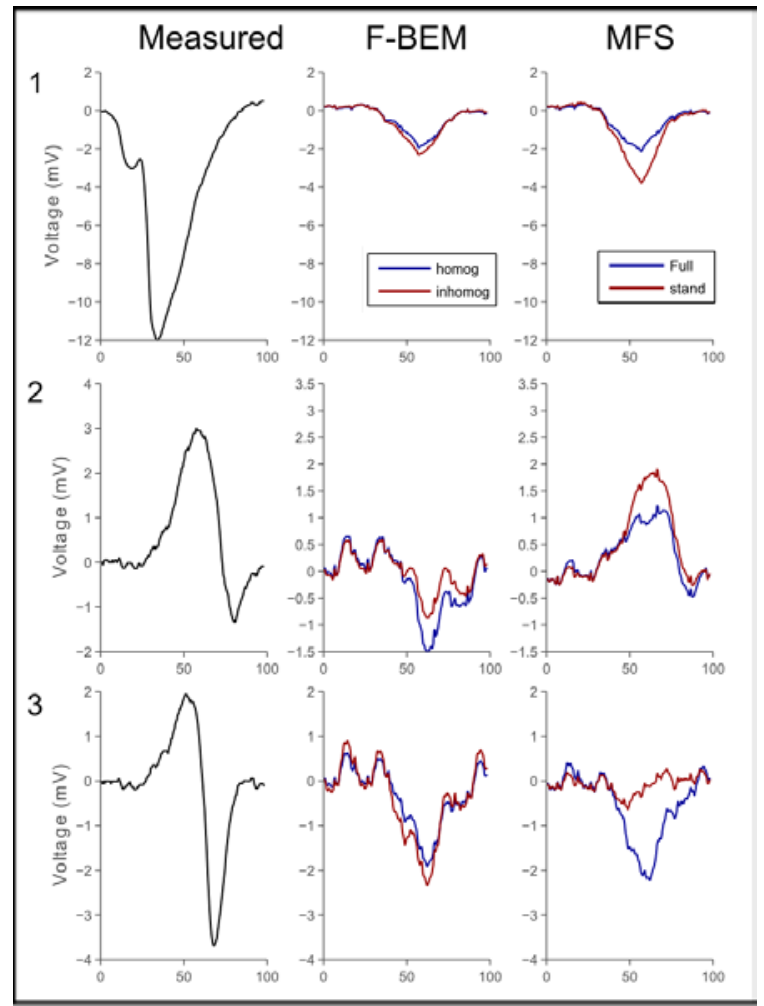

Fig 1. Measured and reconstructed EGMs during RV epicardial pacing.

The magnitudes of reconstructed EGMs were substantially smaller than recorded (uniformly large RE values). Despite this, the general shape of the EGM waveforms were typically recovered (CC > 0.7 for $~ 50 \%$ of electrodes for all models). The intrinsic deflection was often temporally shifted, and complex waveforms were poorly reproduced (i.e., electrode 1 shows smoothing of the R-wave). For all models, a small number of reconstructed EGMs were inverted $(\mathrm{CC}<0)$, or showed little correspondence with measured data (i.e., electrode 2, F-BEM models, and electrode $3, \mathrm{MFS}_{\text {stand }}$ ).

For some electrodes, the MFS models appeared to improve the magnitude, and morphology of EGMs compared to the F-BEM reconstructions (i.e., electrodes 
1, and 2) and vice-versa in others (i.e., electrode 3). In some of these cases (and for all electrodes shown here), the $\mathrm{MFS}_{\text {Full }}$ EGMs were also notably different from the $M_{\text {stand }}$ model, either improving or deteriorating the results. Overall, there appeared to be no systematic improvement in CC, though potentially a slight improvement in RE with the MFS method compared to the BEM (Fig 2). There was no evident difference between homogeneous or inhomogeneous F-BEM models.
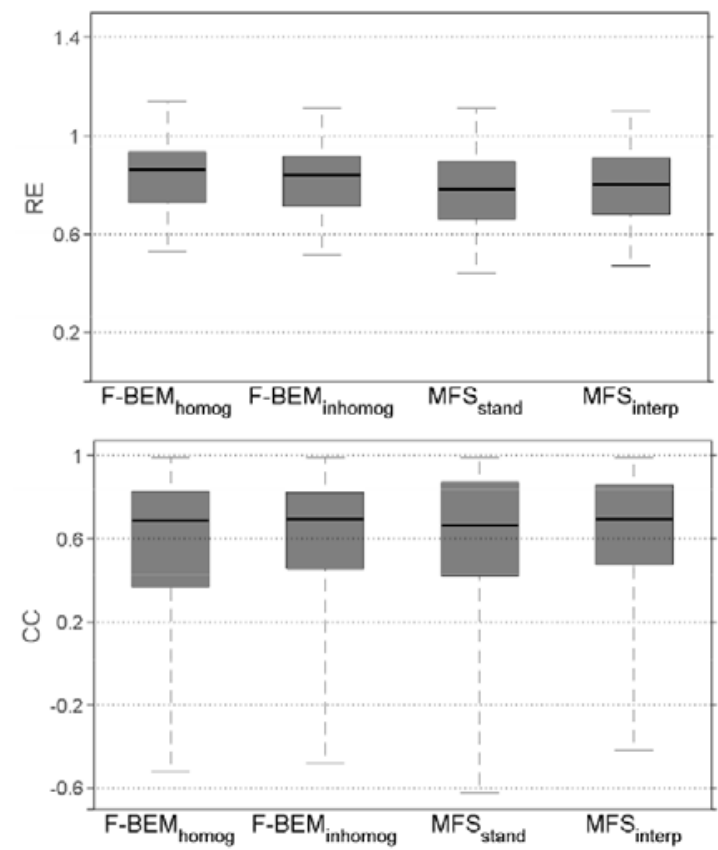

Fig 2. Boxplot distributions of CC and RE computed between all measured and reconstructed EGMs for a RV epicardial pacing sequence.

The results were similar to this case across all activation sequences (Table 1). Here CC and RE were averaged over electrodes for each activation sequence $\left(\mathrm{CC}_{\text {mean }}\right.$ and $\left.\mathrm{RE}_{\text {mean }}\right)$, with the mean $\pm \mathrm{SE}$ presented for the pooled data. There was a statistically significant difference in $\mathrm{RE}_{\text {mean }}$ between the MFS and the F-BEM models $(p<0.001)$. There was no significant difference in $\mathrm{CC}_{\text {mean }}$ among the four models, or in $\mathrm{RE}_{\text {mean }}$ between $\mathrm{MFS}_{\text {full }}$ and $\mathrm{MFS}_{\text {stand, }}$ or between F-BEM $\mathrm{homog}_{\text {and }}$ F$\mathrm{BEM}_{\text {inhomog }}(\mathrm{p}>0.05)$.

Table 1. Summary of comparison between reconstructed and measured EGMs. Results presented as mean \pm SE.

\begin{tabular}{lcc}
\hline & $\mathrm{CC}_{\text {mean }}$ & $* \mathrm{RE}_{\text {mean }}$ \\
\hline${\mathrm{F}-B E M_{\text {homog }}}$ & $0.54 \pm 0.10$ & $0.86 \pm 0.08$ \\
${\mathrm{~F}-B E M_{\text {inhomog }}}$ & $0.55 \pm 0.08$ & $0.87 \pm 0.07$ \\
$\mathrm{MFS}_{\text {Full }}$ & $0.56 \pm 0.09$ & $0.84 \pm 0.09$ \\
MFS $_{\text {stand }}$ & $0.56 \pm 0.08$ & $0.83 \pm 0.09$ \\
${ }^{*} \mathrm{p}<0.05$ F-BEM versus MFS.
\end{tabular}

\subsection{Epicardial activation maps}

AT maps computed from measured and reconstructed potentials are presented in Fig 3 for an RV pacing sequence. The correspondence between measured and reconstructed AT maps is reflected by high CC values. Despite this, total activation duration was $\sim 50 \mathrm{~ms}$ shorter with all reconstructed signals compared to experimental data, and the initial activation sites were $>2 \mathrm{~cm}$ from there true locations. There was little difference F-

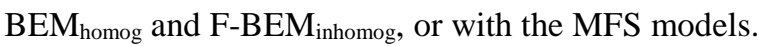

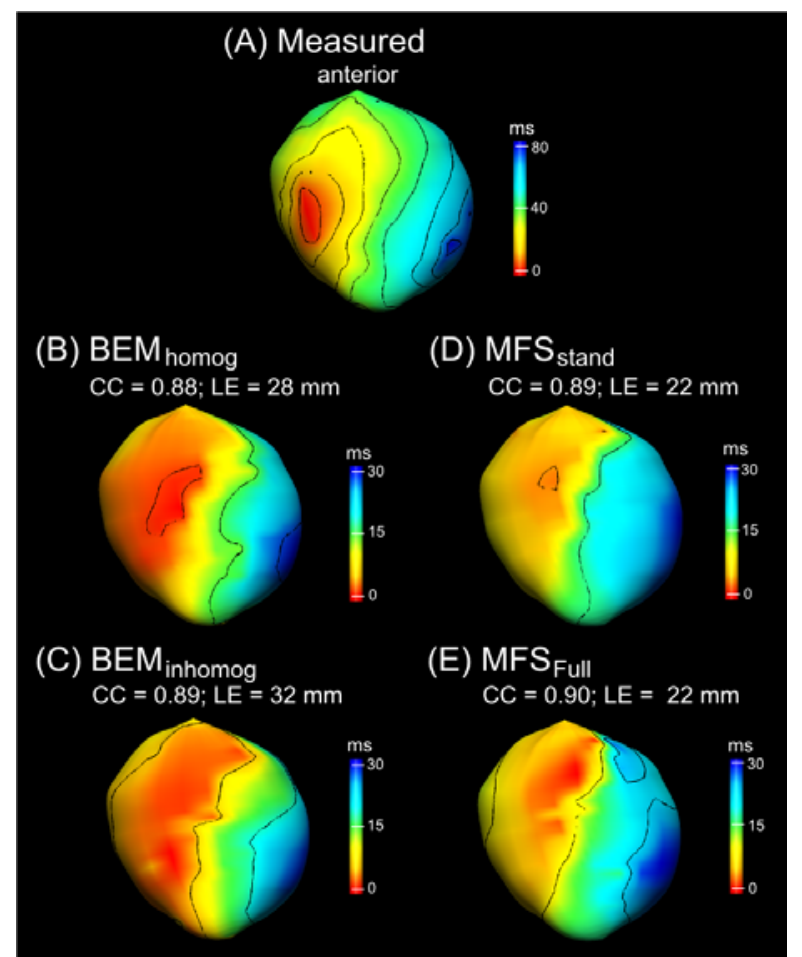

Fig 3. AT maps computed from (A) measured and (BE) reconstructed potentials using BEM and MFS models. Corresponding CC and RE computed between the measured and reconstructed ATs are presented.

Table 2 Summary of comparison between reconstructed and measured ATs. Results presented as mean \pm SE.

\begin{tabular}{lcccc}
\hline & ${ }^{*} \mathrm{CC}$ & $\mathrm{RE}$ & $\mathrm{ADd}$ & ${ }^{*} \mathrm{LE}$ \\
\hline $\mathrm{BEM}_{\text {homog }}$ & $0.73 \pm 0.12$ & $0.48 \pm 0.11$ & $41 \pm 12$ & $27 \pm 22$ \\
$\mathrm{BEM}_{\text {inhomog }}$ & $0.74 \pm 0.22$ & $0.46 \pm 0.09$ & $41 \pm 12$ & $29 \pm 22$ \\
MFS full & $0.78 \pm 0.09$ & $0.46 \pm 0.09$ & $42 \pm 12$ & $18 \pm 22$ \\
MFS $_{\text {stand }}$ & $0.75 \pm 0.09$ & $0.47 \pm 0.09$ & $42 \pm 13$ & $19 \pm 22$ \\
\hline \multicolumn{2}{l}{${ }^{*}<0.05$ F-BEM versus MFS. } & & &
\end{tabular}

These trends were replicated across all activation sequences (Table 2). That is, from CC we can see activation spread was recovered with reasonable accuracy, though total activation duration was substantially underestimated. For all metrics, there was no 
significant difference between BEM $\mathrm{homog}_{\text {and }} \mathrm{BEM}_{\text {inhomog, }}$ or between $\mathrm{MFS}_{\text {full }}$ and $\mathrm{MFS}_{\text {stand }}(\mathrm{p}>0.05)$. Likewise, there was no significant difference in $\mathrm{RE}$ or $\mathrm{ADd}(\mathrm{p}>0.05)$ between BEM and MFS models. However, CC and LE $(p<0.04)$ were significantly better with the MFS models compared to the BEM models

\section{Discussion}

This study demonstrated that inverse epicardial mapping captures the general features of activation, both in terms of EGM morphology and activation spread. However, it substantially underestimates potential magnitudes, activation duration, and initial activation sites are not precisely identified. There was no difference in the results achieved with the inclusion of inhomogeneities and, only minor improvements with an MFS compared to an F-BEM model. Considering this, in addition to the advantage that MFS does not requires meshes, MFS was the preferred approach.

The results presented support the use of noninvasive mapping to complement established ablation therapy. While the magnitudes of EGMs were underestimated (possibly limiting detection of infarcted tissue), qualitative information can be provided on the origin and spread of epicardial activation. Further post-processing of the data could provide further information to clinicians, i.e. phase mapping in atrial tachy-arrhythmias.

Previous work has demonstrated that inhomogeneous structures improved the forward model, but did not eliminate the differences between measured and recorded BSPMs [6]. Here it appears that any improvement gained from the improved forward transfer matrix has been offset by an increase in model complexity. The introduction of greater uncertainty in organ geometries and conductivity values has resulted in an inverse problem that is more illposed. This is supported by previous numerical studies where epicardial potentials were recovered more precisely with inhomogeneous model reconstructions, though these were degraded further by the presence of introduced error [8].

It should be noted that in this study, interpolation was used in order to provide the inputs for both MFS Full $_{\text {and F- }}$ BEM models, which can introduce error into the solutions [9,10]. Indeed, some individual $\mathrm{MSF}_{\text {Full }}$ EGMs were notably less accurate than with the $\mathrm{MFS}_{\text {stand. Despite this, }}$ there was still a significant improvement with the MFS $_{\text {Full }}$ compared to the F-BEM models, and no difference between the $\mathrm{MFS}_{\text {Full }}$ and $\mathrm{MFS}_{\text {stand }}$. Alternative approaches to interpolation [9] may improve F-BEM solutions.

\section{Acknowledgements}

This study was funded, in part, by Programme Grant 09/067 from the Health Research Council of New
Zealand, by the CORDIS3D IRSES-GA-2013-317767 grant and by the French Agence Nationale de la Recherche (ANR-10-IAHU04-LIRYC).

\section{References}

[1] Zhang J, Desouza KA, Cuculich PS, Cooper DH, Chen J, Rudy Y. Continuous ECGI mapping of spontaneous VT initiation, continuation, and termination with antitachycardia pacing. Hear Rhythm. 2012;10(8):1-2.

[2] Cuculich PS, Wang Y, Lindsay BD, Faddis MN, Schuessler RB, Damiano RJ, et al. Noninvasive characterization of epicardial activation in humans with diverse atrial fibrillation patterns. Circ. 2010;122(14):1364-72.

[3] Wang Y, Rudy Y. Application of the method of fundamental solutions to potential-based inverse electrocardiography. Ann Biomed Eng. 2006;34(8):1272-88.

[4] Bradley CP, Pullan AJ, Hunter PJ. Geometric modeling of the human torso using cubic hermite elements. Ann Biomed Eng. 1997;25(1):96-111.

[5] Barr RC, Ramsey M, Spach MS. Relating epicardial to body surface potential distributions by means of transfer coefficients based on geometry measurements. IEEE Trans Biomed Eng. 1977;24(1):1-11.

[6] Bear LR, Cheng LK, LeGrice IJ, Sands GB, Lever NA, Paterson DJ, et al. Forward Problem of Electrocardiography. Circ Arrhythmia Electrophysiol. 2015;8(3):677-84.

[7] Dubois R, Labarthe $S$, Coudière $Y$, Hocini $M$, Haïssaguerre M. Global and directional activation maps for cardiac mapping in electrophysiology. Computing in Cardiology 2012;39:349-52.

[8] Ramanathan C, Rudy Y. Electrocardiographic imaging: II. Effect of torso inhomogeneities on noninvasive reconstruction of epicardial potentials, electrograms, and isochrones. J Card Electrophysiol. 2001;12(2):241-52.

[9] Ghodrati A, Brooks DH, Macleod R. Methods of Solving Reduced Lead Systems for Inverse Electrocardiography. IEEE Trans Biomed Eng. 2007;54(2):339-43.

[10] Bear LR, Potse M, Duchateau J, Zemzemi N, Coudiere Y, Dubois R. Accuracy of lead removal vs linear interpolation in non-invasive electrocardiographic imaging (ECGI). Computimg in Cardiology 2015;42:941-4.

Dr. Laura Bear,

IHU-Liryc, Hôpital Xavier Arnozan, Avenue du Haut Lévèque, 33600 Pessac, France.

laura.bear@ihu-liryc.fr 